\title{
ON CONVEX SETS IN LINEAR NORMED SPACES
}

\section{TRUMAN BOTTS}

M. Eidelheit has proved $^{1}$ this theorem.

THEOREM. In a linear normed space two convex bodies (that is, convex sets with inner points) having no common inner points are separated ${ }^{2}$ by a plane.

The purpose of this note is to present a quite different and somewhat simpler proof of this result. ${ }^{3}$

It is known ${ }^{4}$ for linear normed spaces that

(1) Through every boundary point of a convex body there passes a plane supporting the body.

A convex cone with the point $x_{0}$ as vertex is defined as a convex body $C$ containing at least one point $x \neq x_{0}$ and such that for each such point $x$ in $C$,

$$
a x+(1-a) x_{0} \in C, \quad a \geqq 0 .
$$

It is easily seen that

(2) Every supporting plane of a convex cone $C$ passes through the vertex $x_{0}$ of the cone.

For, let $L(x)-b=0$, where $L(x)$ is a linear functional and $b$ is a constant, define a plane of support of $C$ passing through a boundary point $y$ of $C$. Suppose for definiteness that

$$
L(x)-b \leqq 0
$$

holds for all points $x$ in $C$. Then since every point of the form $a y+(1-a) x_{0}(a \geqq 0)$ is a boundary point of $C$,

$$
L\left(a y+(1-a) x_{0}\right)-b \leqq 0, \quad a \geqq 0,
$$

Presented to the Society, September 5, 1941; received by the editors May 6, 1941.

${ }^{1} \mathrm{M}$. Eidelheit, Zur Theorie der konvexen Mengen in linearen normierten Räumen, Studia Mathematica, vol. 6 (1936), pp. 104-111.

${ }^{2}$ Two sets are separated by a plane provided they lie in opposite closed halfspaces of the plane.

${ }^{3}$ Added in proof: There has recently been brought to my attention another proof of Eidelheit's theorem by S. Kakutani, Proceedings of the Imperial Academy of Japan, vol. 13 (1937), pp. 93-94. The first part of the present proof is closely related to the first part of Kakutani's proof.

${ }^{4}$ See S. Mazur, Über konvexen Mengen in linearen normierten Räumen, Studia Mathematica, vol. 4 (1933), p. 74. 
whence by the linearity of $L$

$$
a(L(y)-b)+(1-a)\left(L\left(x_{0}\right)-b\right) \leqq 0, \quad a \geqq 0 .
$$

But $L(y)-b=0$. Hence

$$
(1-a)\left(L\left(x_{0}\right)-b\right) \leqq 0, \quad a \geqq 0,
$$

and since the factor $(1-a)$ can change sign, we must have

$$
L\left(x_{0}\right)-b=0 .
$$

Let $K_{1}$ and $K_{2}$ be convex bodies with no common inner points. Let $x_{1}$ and $x_{2}$ be inner points of $K_{1}$ and $K_{2}$, respectively. There are unique boundary points $x_{1}^{\prime}$ and $x_{2}^{\prime}$ of $K_{1}$ and $K_{2}$ on the segment $\overline{x_{1} x_{2}}$. Consider the (perhaps degenerate) segment

$$
L \equiv \overline{x_{1}^{\prime} x_{2}^{\prime}}
$$

Let $L_{1}$ be the set of all points $x \in L$ such that no segment joining $x$ to an inner point of $K_{1}$ contains an inner point of $K_{2}$. The set $L_{1}$ is non-empty, since $x_{1}^{\prime} \in L_{1}$. Furthermore, since the complement of $L_{1}$ in $L$ is clearly open in $L, L_{1}$ is closed in $L$. Likewise, if $L_{2}$ is analogously defined, $L_{2}$ is a non-empty set closed in $L$.

Suppose there exists a point $x \in L-\left(L_{1}+L_{2}\right)$. Then it is possible to join $x$ to inner points $y_{1}$ and $y_{2}$ of $K_{1}$ and $K_{2}$ such that there exist inner points $z_{1}$ and $z_{2}$ of $K_{1}$ and $K_{2}$ lying on the (open) segments

$$
\overline{x y_{2}}, \quad \overline{x y_{1}},
$$

respectively. But then the segments

$$
\overline{y_{1} z_{1}}, \quad \overline{y_{2} z_{2}}
$$

intersect in a point $z$ which is interior to both $K_{1}$ and $K_{2}$, contradicting the hypothesis. Hence the supposition that $L-\left(L_{1}+L_{2}\right)$ is non-empty is false, and $L=L_{1}+L_{2}$.

It now follows from the connectedness of $L$ that there exists a point $x_{0} \in L_{1} \cdot L_{2}$. Let $C_{1}$ and $C_{2}$ be the sets consisting of all points on rays from $x_{0}$ through the inner points of $K_{1}$ and $K_{2}$, respectively.

(3) The sets $C_{1}$ and $C_{2}$ are convex cones having no common points except $x_{0}$.

The fact that $C_{1}$ and $C_{2}$ are convex cones is evident. If $C_{1}$ and $C_{2}$ had a common point $y \neq x_{0}$, then on the ray from $x_{0}$ through $y$ there would have to be a point interior to $K_{1}$ and a point interior to $K_{2}$, contradicting $x_{0} \in L_{1} \cdot L_{2}$. 
Take the point $x_{0}$ to be the origin $\theta$. Let $C_{5}^{-}$denote the reflection in the point $\theta$ of the cone $C_{1}$ : that is, $C_{1}^{-}$is the set of all points of the form $-x, x \in C_{1}$. The set $C_{1}^{-}$is a convex cone with $\theta$ as vertex. Let $C$ denote the set of all points of the form

$$
a x+(1-a) y,
$$

where $x \in C_{2}, y \in C_{1}^{-}$, and $0 \leqq a \leqq 1$.

(4) The set $C$ is a convex cone with $\theta$ as vertex. Furthermore, $C$ contains no point interior to $C_{1}$.

The first statement in (4) is easily verified. That the second holds is seen as follows. Any point of $C$ is of the form

$$
z=a x+(1-a) y, \quad x \in C_{2}, y \in C_{1}^{-}, a \in[0,1] .
$$

Let $x_{1}$ be an inner point of $C_{1}$. From (3) it follows that on the segment $\overline{x x_{1}}$ there is a boundary point $x_{1}^{\prime}$ of $C_{1}$. Now by (1) $C_{1}$ has a supporting plane $H_{1}$ passing through $x_{1}^{\prime}$. Since the point $x_{1}^{\prime}$ of $H_{1}$ is on the segment $\overline{x_{1} x}$, the points $x_{1}$ and $x$ lie in opposite closed half-spaces of $H_{1}$. Since $x_{1}$ is interior to $C_{1}, x_{1}$ lies in the half-space of $H_{1}$ containing $C_{1}$. Hence the set $C_{1}$ and the point $x$ lie in opposite closed half-spaces of $H_{1}$; that is, $C_{1}$ and $x$ are separated by $H_{1}$. By (2) $\theta \in H_{1}$, so that $C_{1}$ and $y$ are separated by $H_{1}$. Hence $H_{1}$ separates the sets $C_{1}$ and $\overline{x y}$, and the point $z$, which is contained in $\overline{x y}$, cannot be interior to $C_{1}$.

From (4) and (1) it now follows that $C$ has a plane of support $H$ passing through the point $\theta$. But $H$ is then a plane of support of $C_{2}$. Likewise it is a plane of support of $C_{1}^{-}$and hence of $C_{1}$. Since $C_{\overline{1}}^{-}$and $C_{2}$ are on the same side of $H, C_{1}$ and $C_{2}$ are separated by $H$. Therefore $K_{1}$ and $K_{2}$ are separated by $H$.

UNIVERSITY OF VIRGINIA 